\title{
Die Theorie der Waldreinertragswirtichaft.
}

Som (Beh. Dherforftrat $a$. D. Freł in Darmitabt.

Șm Ditoberbeft ber 2tllgemeinen Forift= und Sagozeitung von 1904

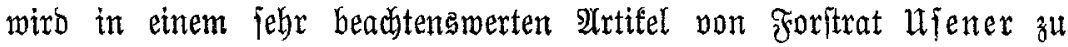
Straß̧burg i. E. wieber einmal auf bie Syaltlofitgeit ber Grand olagen, atf welchen bie Theorie Der \$odenreinertragswirtichaft aufgebaut ijt, hin= gewiejert. Es wiro in braitifcher $\mathfrak{B e i f e}$ als eine "vergebliche Mühe" be= zeichnet, burh eine (Słleichung bie eine Unbetannte (den Sobenmert) aus ber anberen lunbefannten (bem Zinsfups) berechnen zu mollen, und vom Stanopinft des Praftifers btefe Theorie mit ben ssorten verurteilt:

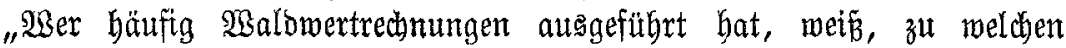

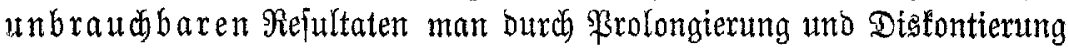
ber Der Gsegenwart entiprechenden Merte gelangt."

Sin "Bemerfingen”, welche biejem $\mathfrak{2}$ (rtifel von Frofeffor Dr. $\mathfrak{B}$ imme= nauer zu Gitęen angefügt woroen find, erflärt fich leb̧terer — obwohl er eint $2 \mathfrak{n}$ hänger ber Bobenreinertraggtheorie - mit bem von bem $\mathfrak{B e r}=$ faffer verfodtenen $\mathfrak{B}$ irtichaftszicl: ber Sinhaltung hoher Umtriebszeiten und

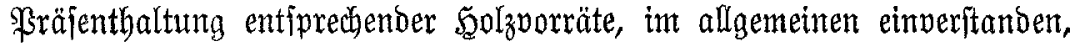

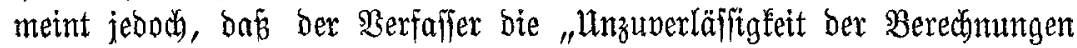

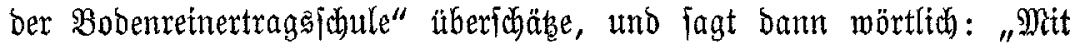

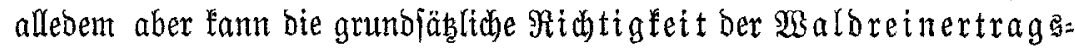
theorie nidet erwiejen merben."

Diejer 2lusfpruth gibt uns Beranlafinng, auf unjere "Mlethobe ber Iaujhwwerte" (Berlin, Berlag von Şulius Springer, 1888) Ginzumeifen,

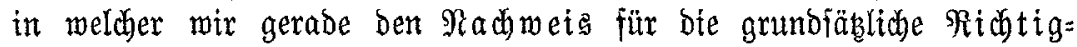
feit Der $\mathfrak{S a l d r e i n e r t r a g s l e b r e ~ e r b r a h t ~ h a b e n . ~}$

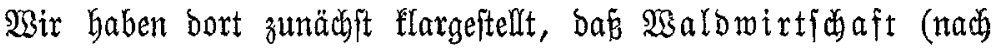
Analogie aller anberen gemerblicben Betriebe) nur betrieben werben

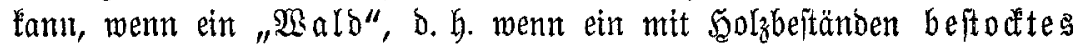

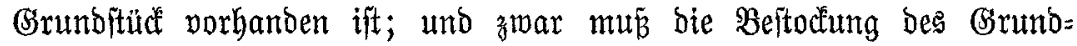
ftüdf's eine berartige jein, Dás ber auf bemjelben jährlich erfolgende, in

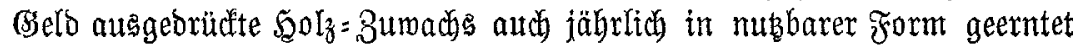
werben Lann, ohne daz ber vorbandene, zum ununterbroctenen Bemerbe= betrieb erforberfidje $\mathfrak{S}_{0} l_{z}=$ Borrat, jeinem (Seldwerte nach, gemindert wird. Wir Gaben bort nachgewiejen, bá biejer Bebingung entjprodben wird, wenn ber $23 e r t$ bes auf biejem Grundftül ftodtenden $F_{2}$ olzoorrates an

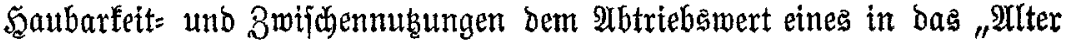




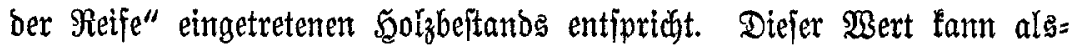
bann burch bie einfache Formel ausgebrüdt merben:

$$
\mathrm{N}_{\mathrm{x}}=\frac{\mathrm{x} \cdot \mathrm{r}_{\mathrm{x}}}{2}
$$

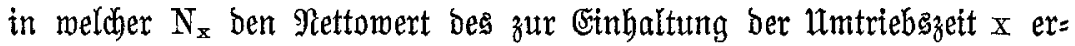

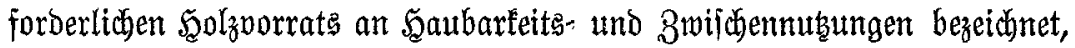

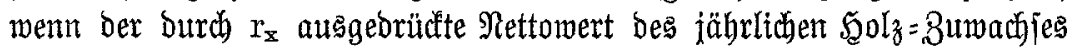
an Šaubarfetts= und 3mijbenmukungen alliährlich geerntet werden foll.

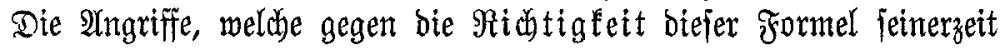
gerichtet worben find, baben wir im Maiheft ber 2ulgenteinen Forit= unt Jagdzeitung von 1889, bezm. im Jultheft ber 3eitfobrift für Forft: und

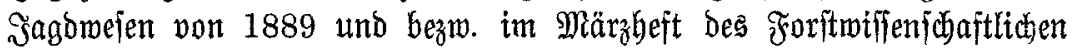
Centralblattes von 1891 zurüdfgemiejen, uno müffen bier - um $\mathfrak{B i e b e r =}$ holungen zu vermeiden - auf bie bezïglichen, unter bem $\mathfrak{i t t e l}$ unierer Schrift: "Die Methode ber Tauffiberte" veröffentlidbten Mbhandlungen vermeifen. Mix wollen biex mur nodymals betonen, dás wir bei allen

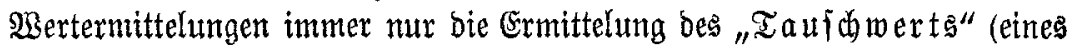
mit "Berfaufsmert", "Marftpreis", "Rurswert", "gemeinem pder reellem

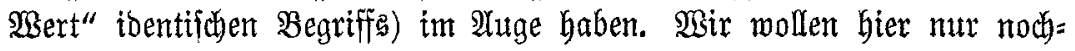
mals hervorbeben, bá unjere Rechnungsmethode allein ben Bwect ver= folgt, praftifich brauchbare Zablenausbrülfe für bie Bertbemeffung von Balbungen zu geminnen; aber wir legen zugleich bas gröb̈te Esemidat barauf, in theoretific) und mathematij

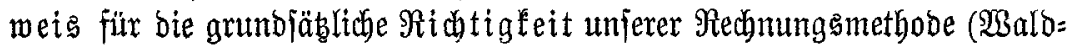
reinertraggtheorie) erbracht zu haben.

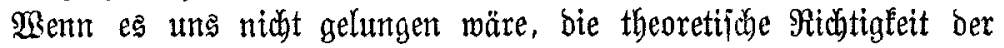
Baldreinertraggmirtidaft nadzumeijen, dann mürbe Frofeffor Dr.

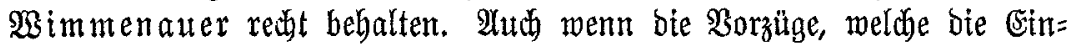
baltung hoher $\mathfrak{U}$ mtriebsgeiten uno bie Präjenthaltung groper ftodfender Szolzoorräte beitien, ala Ergebniffe ber $\mathfrak{B a l b r e i n e r t r a g s t h e o r i e ~ a n e r t a n n t ~}$ werben follten, jo lönnte bod) "mit alledem" für bie "situtigfeit" jener Iheorie nidbt eingetreten werben.

übrigens baben wir - abgejeben von bem pofitiven Bemeis für

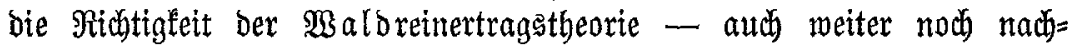

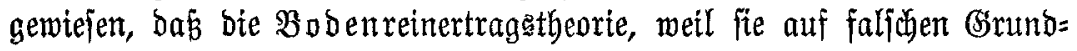

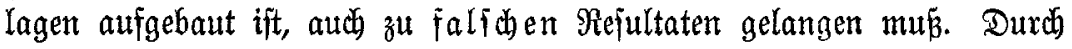

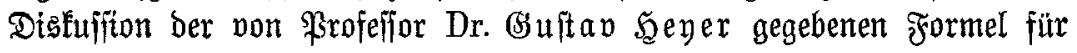
Den "Bobenerwartungswert":

$$
B_{e}=\frac{A_{n}+D_{a} \cdot 1,0 p^{n-a}+\ldots \ldots-c \cdot 1,0 p^{u}}{1,0 p^{u}-1}-V,
$$


weldhe fich auch in ber einfacheren Frorm (cf. unjere int Dezemberbeft

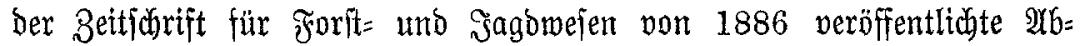

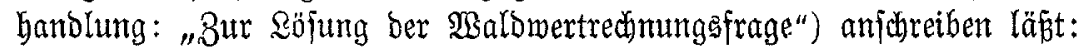

$$
\mathrm{B}_{\mathrm{e}}=\frac{\mathrm{R}-\mathrm{S}}{1,0 \mathrm{p}^{\mathrm{u}}-1}
$$

haben mix nachgemiejen, bas ber ats biejer Formel fid beredinende "Bobenermartungsmert" jeben beliefigen poftiven und negativen Bert von $\varnothing$ bis $\infty$ annehmen $\mathfrak{k} a n$, baj baher bieje formel zur $\mathfrak{E} r=$ mittelung des wirtlichen (reellen) Sobenwerts, ber niemals negativ, fondern

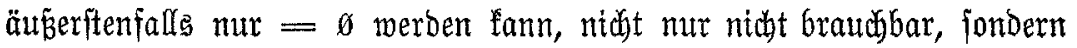
offenbar logifich und mathematifich unriditig ift, und baourch nichts an

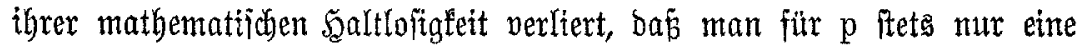

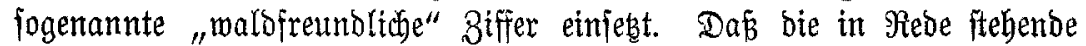
Formel für den "Bobenermartungsmert" feine mathematifiche Dishuffion verträgt, rübrt - wie auf Seite 49 bis 52 unjerer oben erwähnten Săhift näber ausgefübrt wiro - baher, bá fie auf faljober (Snundlage aufgebaut

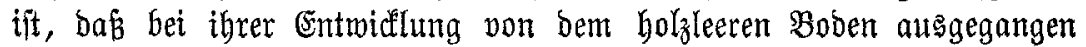

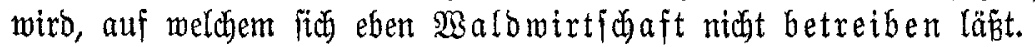

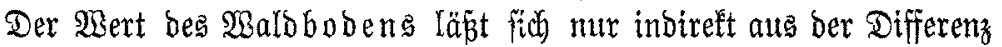

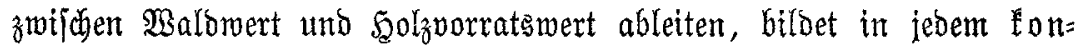

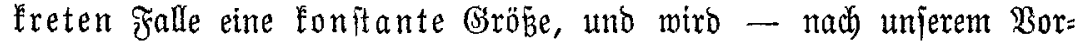
joblag auf Seite 85 unjerer "Methode ber Taujdberte" - am eimjadften

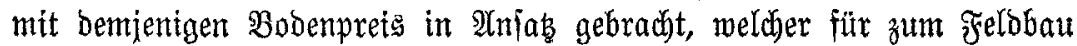
nur jobledft geeigneten, baher ber Şolzzudgt gewiometen Boben in ber be= treffenden (Gemarfung bezaldt $\mathfrak{u}$ werbent pflegt. $2 \mathfrak{d u d}$ baben wix wieber=

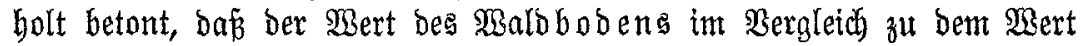
Des zum $\mathfrak{B a l b m i r t i d j a f t a b e t r i e b ~ e r f o r d e r l i c h e n ~ f t o c k e n d e n ~ S o l z o o r r a t s ~ ( v o r a u s = ~}$

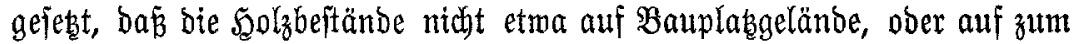

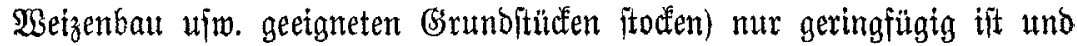

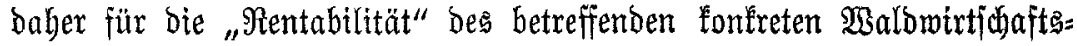
betriebs nux wenig ins Gewidyt fallt.

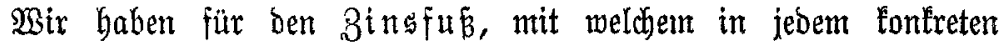
Falle ber betreffende Walowirtiouftsbetrieb fich renttert, bie allgemeine Formel entrictelt:

$$
\begin{aligned}
0,0 p & =\frac{r_{x}}{B_{u}+N_{x}} \\
\text { pber } p & =\frac{100 \cdot r_{x}}{B_{u}+N_{x}}
\end{aligned}
$$

in welder $x, N_{x}$ und $r_{x}$ bie oben erwähnten $\mathfrak{3 e r t e , ~} B_{u}$ ben Tauldjwert

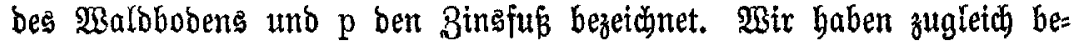




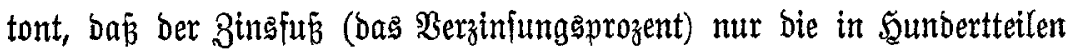

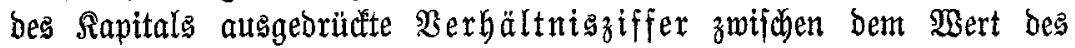
Sahresertrags eines Sapitals und bem $\mathfrak{2}$ Bert bes Rapitals felbft baritellt, und burch Diafujfiton biejer Formel nachgemiejen, da bis biefelbe für alle nur bentbaren $\mathfrak{B s e r t e}$ von $\mathrm{r}_{\mathrm{x}}, B_{\mathrm{a}}$ und $\mathrm{N}_{\mathrm{x}}$ ftets braudbare, mit ben realen Berhältniffen in Übereinftimmung itebende Rejultate liefert, eint

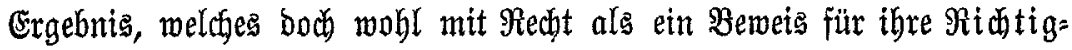
feit angejehen merben barf.

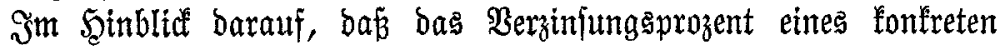

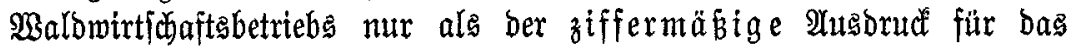

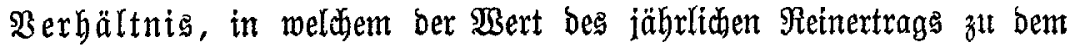
Sapitalwert bes betreffenden $\mathfrak{B a l b e s}$ fteht, zlt betrahten ît, lanm boch

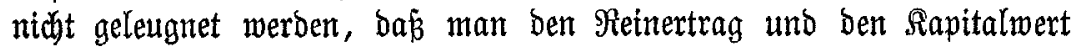

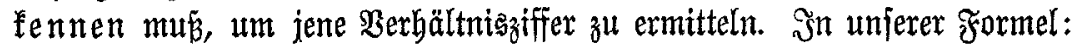

$$
0,0 \mathrm{p}=\frac{\mathrm{r}_{\mathrm{x}}}{\mathrm{B}_{\mathrm{u}}+\mathrm{N}_{\mathrm{x}}}
$$

fint baher auch $r_{x}$ und $N_{x}$ befannte, in jebem fonfreten Falle aus ber

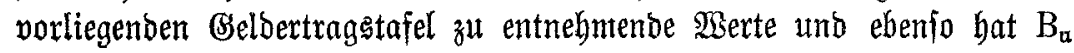
einen - am einfachiten ben mit Dem ortsüblicben Bobenpreis itberein= ftimmenden - befannten 2 Sert, jo baß mit Şilfe biefer Formel fich in jebem fonfreten Fall bas von vornherein anbefannte Şerziniungs:

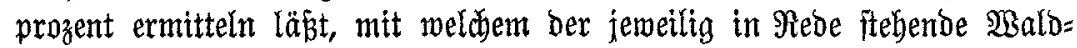

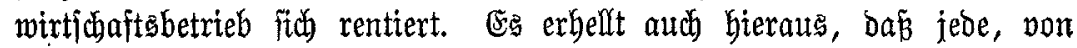

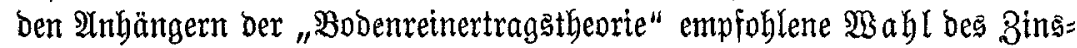
funes - mag biefe mun "maldfreunblich" auğfallen, ober nicht - in

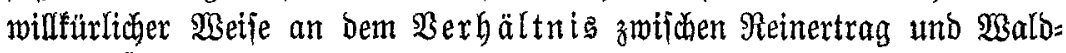

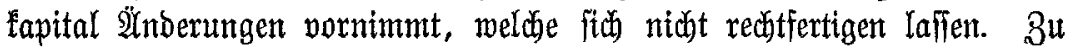
weldhen Trugfbhlitffen man nicht mur bei bem Betrieb bes saldowirt: fidaftsgewerbes, jonbern aud bet jebem anderen Bewerbebetrieb gelangt, wenn man mit einent willfürlich gewäblten und fich unveränbert gleidh bleibenden Zinsfup (ßerzinjungsprozent) rechnet, wie bies ven ben $\mathfrak{A} n=$

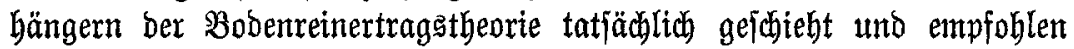
wiro, haben wir in einer unter ber Überibrift: "Sin \$eitrag zur Rebre

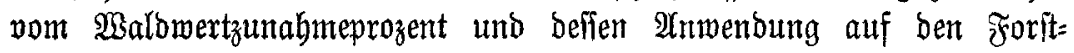

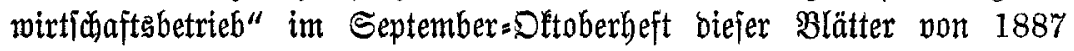
veröffentlichten $\mathfrak{X b h a n b l u n g ~ n a ̈ b e r ~ b a r g e l e g t , ~ a u f ~ w e l d j e ~ w i r ~ u n t s ~ b i e r ~}$ hinzumeijen erlauben.

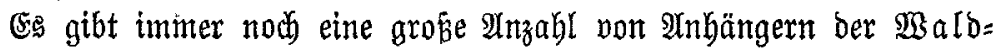
reinertragglebre, weldhe bie Ergebniffe, zut weldhen bieje Sethe ge= langt, zwar billigen und biejelben für praftijo allein braudb bar er= 


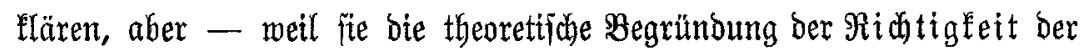
Waloreinertragslehre vermiffen - die fomplizierten Formeln ber Boben= reinertragshehre für mathematifich richtig und unanjectbar balten, wenn

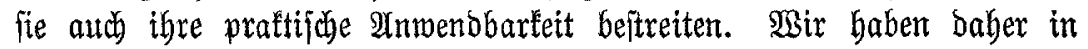
unjerer "Methode ber Taujoberte“ bieje Forme $\mathfrak{i n}$ befämpft uno nad

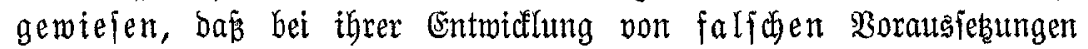
ausgegangen wirb, baj baher - wenn aud noch fo forreft bei ihrem

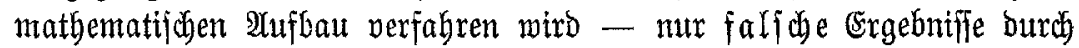
श्tnwendung biejer Formeln erzielt werben fönnen.

Sisir baben bereits in ber "(Sinleitung", auf Seite 1 unjerer oben

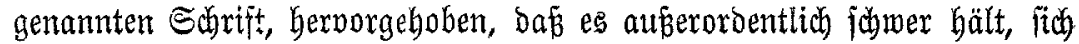

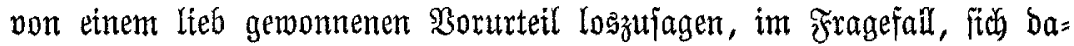

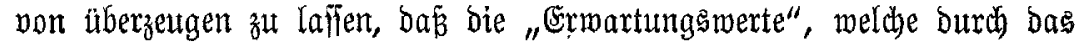
hergebrad) Diste Dontteren von Bufunftserträgen mit einem willfürlid ge=

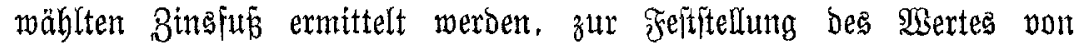
Baldungen völlig unbrauchbar find; wir find in biejer 2 (nfitut burch bie IUsbreitung, welche bie Bobenreinertragshehre bisher erlangt hat, mur

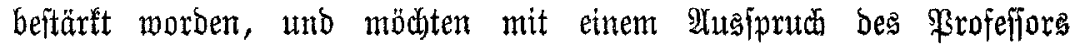

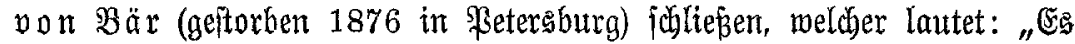

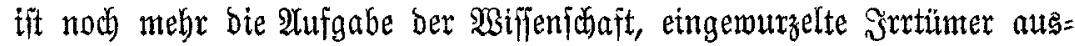
zurotten, als neue $\mathfrak{B a b}$ rheiten zut entbecfen."

Darmitadt, im November 1904.

\section{II. 触tteilumgen.}

\section{Die 5. Gauptwerjammlung des Deutichen forfipereins $3 \mathfrak{u}$ Eijenach 1904.}

Beridterftattung von Forftmeifter Eulefeld=Rauterbad (5̧effen).

Das fajöne Ihüringerland batte mebr benn 500 Teilnehmex zut ber 5. Saauptverjammlung bes beattijent Foritbereins nach dem jagemumwobenen (Eijenad) Gerbetgelndit. $\mathfrak{U m}$ Montag bem 13. September fanben itud bie von Moro und Süo, von Dit uno Beit zum Serzen unjeres Deutichen Baterlandes B̈efommenen zujammen im prädbtigen Saale bes neuerbauten

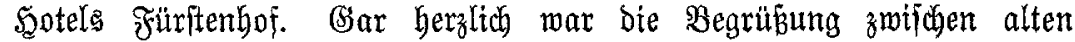

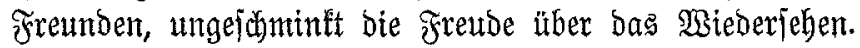

Die Sibungen fanben wom 13. Septentber an im Saale der Erholung

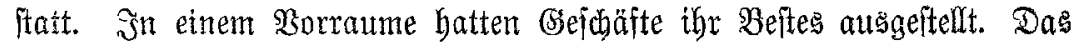
meifte Sintereffe erweclte unitreitig bie altelage aller mögliden forftlicten 